\title{
DO MALNUTRITION, SARCOPENIA AND FRAILTY OVERLAP IN NURSING-HOME RESIDENTS?
}

\author{
G. FAXÉN-IRVING ${ }^{1}$, Y. LUIKING ${ }^{2}$, H. GRÖNSTEDT ${ }^{3}$, E. FRANZÉN ${ }^{4}$, Å. SEIGER ${ }^{5}$, S. VIKSTRÖM ${ }^{6}$, \\ A. $\mathrm{WIMO}^{7}$, A.-M. BOSTRÖM ${ }^{8}$, T. CEDERHOLM ${ }^{9}$
}

\begin{abstract}
1. Department of Neurobiology, Care science and Society, Division of Clinical Geriatrics, Karolinska Institutet, Stockholm and Allied Health Professionals, Functional Area Clinical Nutrition, Karolinska University Hospital, Sweden; 2. Danone Nutricia Research, Utrecht, the Netherlands; 3. Stockholms Sjukhem R\&D unit, Stockholm; Allied Health Professionals, Functional Area Occupational Therapy \& Physiotherapy, Karolinska University Hospital, Stockholm, Sweden; 4. Stockholms Sjukhem R\&D unit, Stockholm, Department of Neurobiology, Care science and Society, Division of physiotherapy, Karolinska Institutet, Stockholm \& Allied Health Professionals, Function Area Occupational Therapy \& Physiotherapy, Karolinska University Hospital, Stockholm, Sweden; 5. Department of Neurobiology, Care Science and Society, Division of Clinical Geriatrics, Karolinska Institutet,

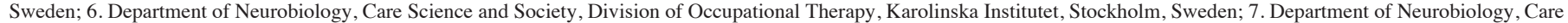
Science and Society, Division of Neurogeriatrics, Karolinska Institutet, Stockholm, Sweden; 8. Stockholms Sjukhem R\&D unit, Stockholm, Department of Neurobiology, Care science and Society, Division of nursing, Karolinska Institutet, Stockholm, and Theme Aging, Karolinska University Hospital, Stockholm, Sweden; 9 . Department of Public Health and Caring

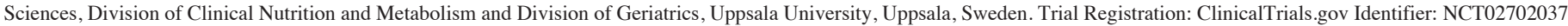
Corresponding author: Gerd Faxén-Irving, Department of Neurobiology, Care science and Society, Division of Clinical Geriatrics, Karolinska Institutet, Stockholm and Allied Health Professionals, Functional Area Clinical Nutrition, Karolinska University Hospital, Sweden, gerd.faxen.irving@ki.se
\end{abstract}

\begin{abstract}
Objectives: To study the prevalence and overlap between malnutrition, sarcopenia and frailty in a selected group of nursing home $(\mathrm{NH})$ residents. Design: Cross-sectional descriptive study. Setting: Nursing homes (NH). Participants: 92 residents taking part in an exercise and oral nutritional supplementation study; $>75$ years old, able to rise from a seated position, body mass index $\leq 30 \mathrm{~kg} / \mathrm{m}^{2}$ and not receiving protein-rich oral nutritional supplements. Measurements: The MNA-SF and Global Leadership Initiative on Malnutrition (GLIM) criteria were used for screening and diagnosis of malnutrition (moderate or severe), respectively. Sarcopenia risk was assessed by the SARC-F Questionnaire (0-10p; $\geq 4=$ increased risk), and for diagnosis the European Working Group of Sarcopenia in Older People (EWGSOP2) criteria was used. To screen for frailty the FRAIL Questionnaire (0-5p; 1-2p indicating pre-frailty, and $>3 p$ indicating frailty), was employed. Results: Average age was 86 years; $62 \%$ were women. MNA-SF showed that $30(33 \%)$ people were at risk or malnourished. The GLIM criteria verified malnutrition in $16(17 \%)$ subjects. One third $(n=33)$ was at risk for sarcopenia by SARC-F. Twenty-seven (29\%) subjects displayed confirmed sarcopenic according to EWGSOP2. Around $50 \%(\mathrm{n}=47)$ was assessed as pre-frail or frail. Six people $(7 \%)$ suffered from all three conditions. Another five $(5 \%)$ of the residents were simultaneously malnourished and sarcopenic, but not frail, while frailty coexisted with sarcopenia in $10 \%(n=9)$ of non-malnourished residents. Twenty-nine (32\%) residents were neither malnourished, sarcopenic nor frail. Conclusions: In a group of selected NH residents a majority was either (pre) frail $(51 \%)$, sarcopenic $(29 \%)$ or malnourished $(17 \%)$. There were considerable overlaps between the three conditions.
\end{abstract}

Key words: Nursing home, older person, malnutrition, frailty, sarcopenia.

J Frailty Aging 2021;10(1)17-21

Published online August 12, 2020, http:/ / dx.doi.org/10.14283/jfa.2020.45

\section{Introduction}

Malnutrition and sarcopenia, commonly occurring in older adults, are associated with negative outcomes (1). Loss of muscle mass and function combined with poor nutrition contributes to an increased risk of frailty; i.e. a state of vulnerability and decreased resilience against stressors (2). Estimated prevalence of physical frailty in the community is around $15 \%$ and $25 \%$ in adults aged $>65$ years and $>85$ years, respectively (3). A review and meta-analysis found frailty to be a significant predictor of all-cause mortality in older $\mathrm{NH}$ residents (4).

The prevalence of malnutrition and risk of malnutrition in NH residents depends on multiple factors, including the tools and criteria for assessment used. Recently the Global Leadership Initiative for Malnutrition (GLIM) suggested a two-step process starting with screening for malnutrition and Received March 6, 2020 Accepted for publication May 28, 2020 then assessment for diagnosis and grading the severity of malnutrition (5).

Sarcopenia, i.e. loss of muscle strength and mass, occurs with aging and is accelerated by inactivity and disease. Sarcopenia leads to impaired ability to perform activities of daily living (ADL), i.e. walking, toileting, eating and socializing, and subsequently results in increased dependence (6). In addition, it increases the risk of falls and pressure ulcers (7). According to a recently published systematic review and meta-analysis, using the European Working Group of Sarcopenia in Older People (EWGSOP) definition from 2010 , the prevalence of sarcopenia was $41 \%$ in older $\mathrm{NH}$ residents (8). The recent EWGSOP2 criteria (9) focuses on low muscle strength as the key characteristic of probable sarcopenia and uses detection of low muscle quantity and quality to confirm the sarcopenia diagnosis. Subsequently, poor physical performance indicates severe sarcopenia. The SARC-F 
DO MALNUTRITION, SARCOPENIA AND FRAILTY OVERLAP IN NURSING-HOME RESIDENTS?

Table 1

Nutritional status, sarcopenia and frailty by gender in selected nursing-home residents

\begin{tabular}{|c|c|c|c|}
\hline & $\begin{array}{c}\text { All } \\
\mathrm{n}=92\end{array}$ & $\begin{array}{c}\text { Men } \\
\mathbf{n}=35(38 \%)\end{array}$ & $\begin{array}{c}\text { Women } \\
\mathrm{n}=57(62 \%)\end{array}$ \\
\hline Age (years) & $86.5 \pm 5.5$ & $86.3 \pm 6.4$ & $85.8 \pm 4.9$ \\
\hline Body mass index (BMI; kg/m²) & $25.4 \pm 3.9$ & $25.6 \pm 3.4$ & $25.3 \pm 4.1$ \\
\hline MNA-SF (0-14p), median (IQR) & $12(11-13)$ & $12(11-13)$ & $12(10-13)$ \\
\hline Normal $\quad(12-14 \mathrm{p}) \mathrm{n}(\%)$ & $62(67)$ & $25(71)$ & $37(65)$ \\
\hline At risk $\quad(8-11 p) \mathrm{n}(\%)$ & $29(32)$ & $9(26)$ & $20(35)$ \\
\hline Malnourished $\quad(0-7 p)$ n (\%) & $1(1)$ & $1(3)$ & $0(0)$ \\
\hline \multicolumn{4}{|l|}{ Malnutrition according to GLIM(5) } \\
\hline Not malnourished & $76(83)$ & $29(83)$ & $47(83)$ \\
\hline Moderate malnutrition, $\mathrm{n}(\%)$ & $12(13)$ & $5(14)$ & $7(12)$ \\
\hline Severe malnutrition, $\mathrm{n}(\%)$ & $4(4)$ & $1(3)$ & $3(5)$ \\
\hline \multicolumn{4}{|c|}{ Sarcopenia according to EWGSOP (9) n (\%) } \\
\hline Not sarcopenic & $25(27)$ & $10(29)$ & $15(26)$ \\
\hline Probable sarcopenia (only) & $40(44)$ & $14(40)$ & $26(46)$ \\
\hline Confirmed sarcopenia (only) & $3(3)$ & $1(3)$ & $2(3)$ \\
\hline Severe sarcopenia (only) & $24(26)$ & $10(29)$ & $14(25)$ \\
\hline SARC-F (0-10p), median (IQR) & $2.5(4)$ & $3(5)$ & $2(4)$ \\
\hline No risk of sarcopenia $(<4 p) n(\%)$ & $59(64)$ & $22(63)$ & $37(65)$ \\
\hline Risk of sarcopenia ( $\geq 4 \mathrm{p}) \mathrm{n}(\%)$ & $33(36)$ & $13(37)$ & $20(35)$ \\
\hline FRAIL (0-5 p), median (IQR) & $1(2)$ & $1(2)$ & $0(2)$ \\
\hline Robust (0 p), n (\%) & $45(49)$ & $16(46)$ & $29(51)$ \\
\hline Pre-frailty (1-2 p), n (\%) & $35(38)$ & $14(40)$ & $21(37)$ \\
\hline Frailty $\quad(3-5 \mathrm{p}), \mathrm{n}(\%)$ & $12(13)$ & $5(14)$ & $7(12)$ \\
\hline
\end{tabular}

Questionnaire was developed to facilitate screening in clinical practice, and it shows a strong capacity to predict poor physical performance and muscle function in older adults (10).

Malnutrition, sarcopenia and frailty frequently interact and coexist in older people. The main objectives of this study were to determine the prevalence of these three catabolic conditions in a selected group of NH residents, and to assess how they overlap. Moreover, we wanted to apply the recently accepted screening and diagnostic tools for sarcopenia and malnutrition in a NH-setting.

\section{Material and methods}

This report is based on baseline data from the Older People Exercise and Nutrition (OPEN) study, a two-arm randomized controlled trial performed in $\mathrm{NH}$ at two municipalities in the Stockholm area (Sweden) (11). Out of 120 residents participating in the OPEN study, 92 had complete data at baseline regarding nutritional status, sarcopenia and frailty and were analyzed in this cross-sectional study.

\section{Participants}

Inclusion criteria for participation were age $\geq 75$ years and ability to rise from a seated position. Exclusion criteria were $\mathrm{BMI}>30 \mathrm{~kg} / \mathrm{m}^{2}$, use of protein-rich oral nutritional supplements, severe dysphagia, tube feeding, bedridden, severe kidney disease, terminal stage of life, and inability to give informed consent. Two clinically experienced physiotherapists from the research group performed the data collection. 


\section{THE JOURNAL OF FRAILTY \& AGING}

\section{Study design and procedures}

Occurrence of malnutrition was assessed in a two-step procedure starting with screening as suggested by the GLIM consortium (5). For screening, the Mini Nutritional Assessment Short Form (MNA-SF) $(0-14 ; 12-14=$ normal nutritional status; $8-11=$ at risk for malnutrition; $0-7=$ malnourished) was used. The diagnosis of malnutrition was set according to the GLIM format that requires at least one phenotypic criterion; i.e. weight loss, underweight or low muscle mass, combined with at least one etiologic criterion; i.e. reduced food intake or severe disease burden. Severity of malnutrition grades as Stage 1 (moderate) or Stage 2 (severe) malnutrition (5) according to the degree of aberration of the phenotypic criteria. Underweight was indicated by BMI $<22 \mathrm{~kg} / \mathrm{m}^{2}$, and BMI $<20 \mathrm{~kg} / \mathrm{m}^{2}$ indicated severe malnutrition.

Bioelectrical impedance analysis (BIA) (ImpediMed SFB7) was performed to estimate body composition into fat free mass index (FFMI in $\mathrm{kg} / \mathrm{m}^{2}$ ) and fat mass index (FMI in $\mathrm{kg} / \mathrm{m}^{2}$ ). A FFMI of $17 \mathrm{~kg} / \mathrm{m}^{2}$ for men and $15 \mathrm{~kg} / \mathrm{m}^{2}$ for women were thresholds for reduced muscle mass (5).

Sarcopenia was assessed by the EWGSOP2 algorithm for case-finding, diagnosis and severity determination (9). SARC-F Questionnaire was used in parallel to assess risk of sarcopenia. The SARC-F questions reflect strength, assistance with walking, rise from a chair, climb stairs and accidental falls; ( $0-10 p ; \geq 4=$ increased risk) (10). According to EWGSOP2 sarcopenia was diagnosed as probable by an impaired chair stand test, and subsequently confirmed when combined with low FFMI. The residents performed a modified timed chair stand test with arms folded over the chest or with support from the chair arms or walking aid (11), and considered impaired when $<10$ chair stands in $30 \mathrm{sec}$ ( $<85$ years) or $<8$ chair stands in $30 \mathrm{sec}$ ( $\geq 85$ years) (11). Severity of sarcopenia was graded by using gait speed (in $\mathrm{m} / \mathrm{sec}$, measured over a distance of $10 \mathrm{~m}$ indoors), with a gait speed below $\leq 0.8 \mathrm{~m} / \mathrm{sec}$ as an indicator of severe sarcopenia.

The FRAIL questionnaire $(0-5 p ; 0=$ robust; $1-2=$ pre-frail and $3-5=$ frail) was used to screen for frailty (12).

\section{Statistical analyses}

Data is presented using descriptive statistics, i.e. mean and SD for continuous variables or median and interquartile range (IQR). The Statistica ${ }^{\circledR} 10.0$ software package (Statsoft) Tulsa, OK, USA) was used for the statistical calculations.

\section{Results}

The residents were on average 86 years old (Table 1). A majority suffered from an average of three diagnoses; cognitive and cardiac disorders were most common (data not shown).

Table 1 shows that mean BMI was around $25\left(\mathrm{~kg} / \mathrm{m}^{2}\right)$. BMI $<22$ and $<20$ were found in $19(21 \%)$ and seven $(8 \%)$ of the residents, respectively. BIA revealed a low FFMI $\left(\mathrm{kg} / \mathrm{m}^{2}\right)$ in 17 (49\%) men and 22 (39\%) women.
One third of the residents was assessed by the MNA-SF screening tool to be at risk of malnutrition or malnourished. Subsequently, the GLIM criteria confirmed malnutrition in a total of $16(17 \%)$ of the participants; i.e. 12 and 4 were graded as moderately and severely malnourished, respectively (Table 1).

The SARC-F Questionnaire depicted around 1/3 of the residents to be at risk of sarcopenia. The EWGSOP2 criteria indicated altogether $40(44 \%)$ to have "probable" sarcopenia, while three $(3 \%)$ and $24(26 \%)$ residents had confirmed and severe sarcopenia, respectively (Table 1). One of four was not sarcopenic. Nineteen out of the 33 residents assessed as at risk by SARC-F were diagnosed as probable and 12 as confirmed sarcopenia according to EWGSOP2.

\section{Figure 1}

Prevalence and overlaps of malnutrition, sarcopenia and frailty in a selected group of nursing-home residents.

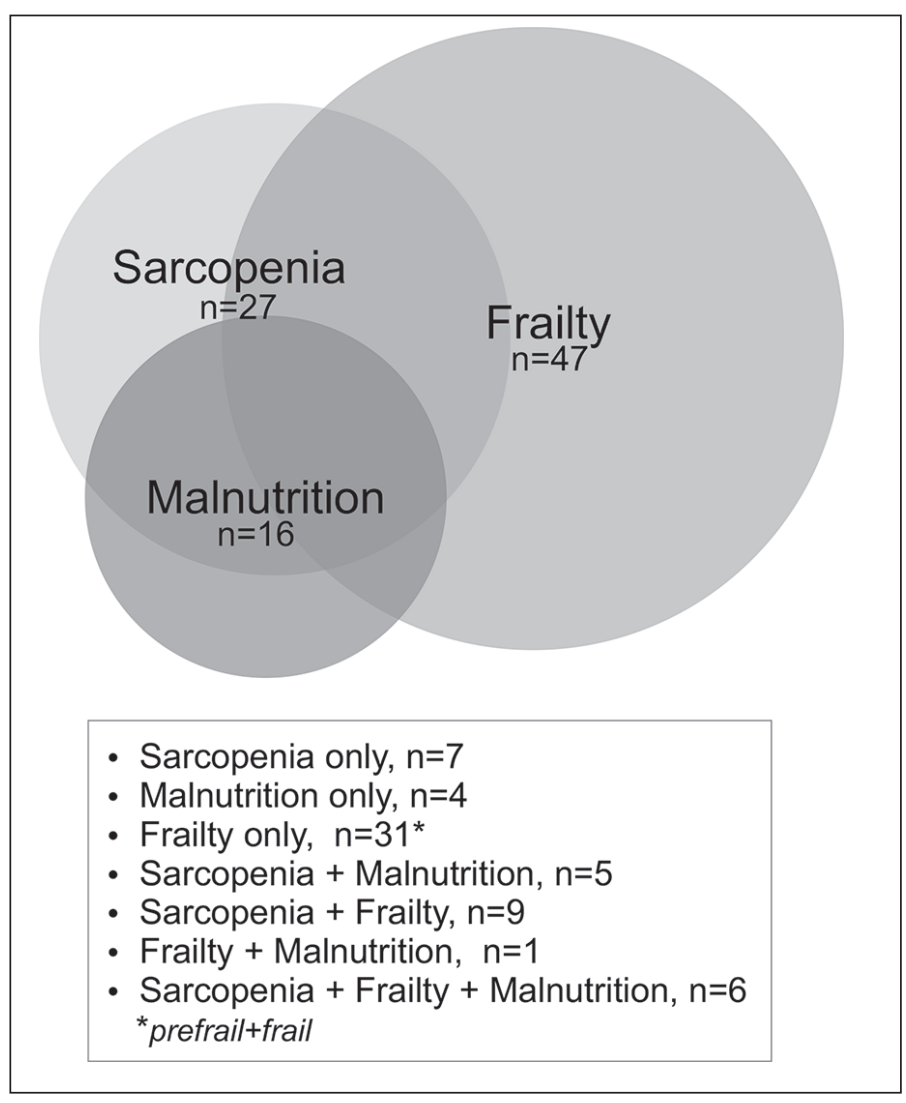

The FRAIL Questionnaire screening indicated a prevalence of pre-frailty (only) and frailty of $38 \%$ and $13 \%$, respectively (Table 1).

The Venn diagram (Fig 1) shows how malnutrition, sarcopenia and prefrail/frailty overlapped. Six (7\%) residents suffered from all three conditions. Malnutrition and sarcopenia co-existed in five non-frail subjects (5\%), and sarcopenia and (pre-)frailty in nine (10\%) non-malnourished subjects. One of the 47 residents identified as pre-frail or frail was also 


\section{DO MALNUTRITION, SARCOPENIA AND FRAILTY OVERLAP IN NURSING-HOME RESIDENTS?}

malnourished, but not sarcopenic. Twenty-nine (32\%) residents were neither malnourished, sarcopenic nor frail.

\section{Discussion}

The aim of this paper is to present prevalence and overlap of malnutrition, sarcopenia and frailty in a selected group of $\mathrm{NH}$ residents and to apply the recently accepted criteria to screen and diagnose sarcopenia (EWGSOP2) and malnutrition (GLIM) in a NH setting. Almost one-third of the residents was sarcopenic (confirmed or severe) (Table 1), one out of five malnourished and half were pre-frail or frail. About one in five displayed an overlap between sarcopenia and malnutrition, in line with a recent report (8). Frailty and sarcopenia showed overlap in one of ten, also in line with a previous report (13). Pre-frailty and frailty overlapped with malnutrition in seven persons; i.e. six were also sarcopenic.

Regarding SARC-F, there was a good agreement between the number of residents screened as at risk of sarcopenia, and those diagnosed as probable and confirmed sarcopenia according to EWGSOP2.

Among the one third $(n=30)$ of the participants who were assessed as being at least at risk of malnutrition according to MNA-SF, about half were diagnosed as malnourished according to the GLIM criteria. Thus, malnutrition was confirmed in altogether $17 \%$ of the residents.

The FRAIL Questionnaire identified close to half of the participants as pre-frail, but only 12 persons (13\%) as frail. This result may indicate that the study group was more robust than the average $\mathrm{NH}$ population.

The ICFSR international expert group recently published guidelines for identification and management of physical frailty and sarcopenia. To treat sarcopenia it is recommended to use resistance-based physical activity and to consider protein-rich oral nutritional supplementation/or protein-rich diet even in the older population living in NH (14). To manage frailty, a multicomponent physical activity program including resistancebased training and protein/energy supplementation (in case of weight loss or undernutrition) is recommended (3).

There are limitations of the study that need to be considered. One is that the selection of NH-residents was based on the capacity to take part in an intervention study, thus reducing the generalizability of the results. Another potential limitation is that we used a modified 30-s timed chair stand; e.g. the participants were allowed to use the upper extremities for support when rising from the chair. This modification may ensure that individuals with low physical function can complete the test and to eliminate the floor effect demonstrated with other sit-to-stand protocols. This chair stand protocol also deviates from the timed five chair stands that EWGSOP recommends, and that is also affected by floor effects in frail sarcopenic older people.

We may conclude that even among a group of fairly robust $\mathrm{NH}$ residents, two thirds suffered from any of the three catabolic conditions sarcopenia (confirmed or severe) (29\%), malnutrition $(17 \%)$ and pre-frailty/frailty $(51 \%)$. There were substantial overlaps between malnutrition and sarcopenia and between frailty and sarcopenia. We suggest that screening and diagnosis of these three conditions should be integrated in $\mathrm{NH}$ care and should be followed by intervention and monitoring.

Funding: The study was financially supported by Danone Nutricia Research. Representatives from Nutricia have been involved in the study design, but the company was not involved in data collection and analyses. The final interpretation of the study results, review, and decision to submit the manuscript was performed by independent researchers with no affiliation to the funding source. The study is also funded by Gamla Tjänarinnor.

Authors' contributions: Gerd Faxén-Irving, Tommy Cederholm, Åke Seiger, Anders Wimo, Anne-Marie Boström were responsible for the design of the protocol and the methodology of the study. Gerd Faxén Irving, Tommy Cederholm, Åke Seiger, Anne-Marie Boström, Erika Franzén, Helena Grönstedt, Yvette C Luiking, Sofia Vikström, Anders Wimo contributed to the writing of the manuscript. All authors read and approved the final manuscript.

Acknowledgements: The authors would like to thank all participating residents and staff in the eight NHs. We are grateful to our Canadian collaborators, Dr. Susan E Slaughter and her research group at University of Alberta, Edmonton, Canada for advice in developing the study protocol. We are also grateful to Dr. Sara Runesdotter for statistical support, Ms. Elin Linde for support regarding data collection and Ms. Frida Eriksson for data management.

Ethical considerations: The study has been approved by the Regional Ethical Review Board in Stockholm, EPN, D no. 2013/1659-31/2, 2015/1994-32 and 2016/1223-32.

Conflict of interest: The authors have received grants from Gamla Tjänarinnor charitable fund, grants from Nutricia Global, during the conduct of the study.

Open Access: This article is distributed under the terms of the Creative Commons Attribution 4.0 International License (http://creativecommons.org/licenses/by/4.0/), which permits use, duplication, adaptation, distribution and reproduction in any medium or format, as long as you give appropriate credit to the original author(s) and the source, provide a link to the Creative Commons license and indicate if changes were made.

\section{References}

1. Vanderwoude MFJ, Alish CJ, Sauer AC, Hegazi RA. Malnutrition-sarcopenia syndrome: is this the future of nutrition screening and assessment for older adults? J Aging Res 2012;1-8.

2. Abellan van Kan G, Rolland YM, Morley JE, Vellas B. Frailty: toward a clinical definition. J Am Med Dir Assoc 2008;9:71-72.

3. Dent E, Morley JE, Cruz-Jentoft AJ, Woodhouse L, Rodriguez-Manas L, Fried LP et al. Physical frailty: ICFSR international clinical practice guidelines for identification and management. J Nutr Health Aging 2019;23(9):771-787.

4. Zhang X, Dou Q, Zhang W, Wang C, Xie X, Yang Y,Zeng Y. Frailty as a Predictor of All-Cause Mortality Among Older Nursing Home Residents: A Systematic Review and Meta-analysis. J Am Med Dir Assoc 2019;20(6):657-663.

5. Cederholm T, Jensen GL, Correia MITD, Gonzalez MC, Fukushima R et al. GLIM Core Leadership Committee, GLIM Working Group. GLIM criteria for the diagnosis of malnutrition - A consensus report from the global clinical nutrition community. Clin Nutr 2018:1-9.

6. Correa-de Araujo R, Hadley E. Skeletal muscle function deficit: A new terminology 


\section{THE JOURNAL OF FRAILTY \& AGING}

to embrace the evolving concepts of sarcopenia and age-related muscle dysfunction. J Gerontol A Biol Sci Med Sci 2014;69:591-594.

7. Landi F, Cruz-Jentoft AJ, Liperoti R, Russo A, Giovannini S, Tosato M et al Sarcopenia: Sarcopenia and mortality risk in frail older persons aged 80 years and older: Results from ilSIRENTE study. Age Ageing 2013;42:203-209.

8. Shen Y, Chen J,Chen X, Hou L, Lin X and Yang M. Prevalence and associated factors of sarcopenia in nursing home residents: A systematic review and meta-analysis. J Am Med Dir Assoc 2019;20:5-13.

9. Cruz-Jentoft AJ, Bahat G, Bauer J, Boirie Y, Bruyère $\mathrm{O}$, Cederholm T et al. Writing group for the European Working Group on Sarcopenia in older people2 (EWGSOP2), and the extended group for EWGSOP2: Sarcopenia: revised European consensus on definition and diagnosis. Age and Ageing 2018;0:1-16.

10. Woo J, Leung J, Morley JE. Validating the SARC-F: A suitable community screening tool for sarcopenia? J Am Med dir Assoc 2014;15:630-634
11. Grönstedt H, Vikström S, Cederholm T, Franzén E, Seiger Å, Wimo A et al. Effect of sit-to-stand exercises combined with protein-rich oral supplementation in older persons. JAMDA 2020 in press.

12. Morley JE, Malmstrom TK, Miller DK: A simple frailty questionnaire (FRAIL) predicts outcomes in middle aged African Americans. J Nutr Health Aging 2012, 16 (7):601-608.

13. Mijnarends DM, Schols J MGA, Meijers J MM, Tan F ES, Verlaan S, Luiking Y $\mathrm{C}$ et al. Instruments to assess sarcopenia and physical frailty in older people living in a community (care) setting: Similarities and discrepancies. J Am Med Dir Assoc 2015;16:301-308

14. Dent E, Morley JE, Cruz-Jentoft AJ, Arai H, Kritchevsky SB, Guralnik J et al International clinical practice guidelines for sarcopenia (ICFSR): Screening, diagnosis and management. J Nutr Health Aging 2018;1-13. 